\title{
A Remote Laboratory Environment for Manipulative Therapy Learning
}

\author{
doi:10.3991/ijoe.v5i2.832 \\ O.P. Dias, T.G. Amaral and V.F. Pires \\ Escola Superior de Tecnologia de Setúbal, Setúbal, Portugal
}

\begin{abstract}
The aim of this paper is to present a remote learning laboratory for physiotherapists. The implementation of such laboratory uses standard PCs, the popular software package Matlab, gloves with sensors and a camera. This system allows the students to do practical experiences at a long distance. They use a glove that measure the force applied by the physiotherapist on the patient. These signals are acquired by the computer student and send it to the school server. Then the teachers can analyze the force applied by the student on the patient. The student must also use a camera that allows to the teacher see the student movements.
\end{abstract}

Index Terms-Distance learning, remote laboratory, physiotherapist, strength sensor.

\section{INTRODUCTION}

With the computer and telecommunications development new possibilities appear to teach at a long distance. In this context e-learning is seen as offering solutions to several challenges. In fact, e-learning provides distance learning and improve the flexibility and quality of learning by: increased access to educational opportunities, individualization of learning and content, targeted skills development, and accommodation of differing learning styles [1].

Many e-learning platforms have been developed. However, many of these tools have been focus on the " $e$ " and less on the "learning". One of the motivating factors for elearners is the possibility of interacting with other colleagues, teachers and has living experiences. In this context, remote laboratories appear with real interactive experiments by using internet connected laboratory equipments. Remote teaching platforms are spreading in the higher education system. Many examples of remote laboratories for different subjects have been developed, such as: electrical [2,3], electronics [4,5], microelectronics $[6,7]$, power systems $[8,9]$, robotics $[10,11]$, systems and control [12, 13]. However, remote laboratories are also very important in the medicine area. In this way, the implementations of some remote labs for such specific subject were developed. As an example there is the ultrasound medical diagnosis [14].

It is known that about $60 \%$ of the patients that seek treatment in the physiotherapy clinics suffer of lumbar pain. To solve or to minimize this type of problem, several therapeutic techniques have been developed, namely the Accessory Passive Intervertebral Movements (APIM) and among them, the Posteroanterior movements (SHOVEL) described by Maitland [15], which are used to evaluate the amplitude of the inter-segmentary displacement of the backbone. With this in mind, a remote laboratory based on an Auxiliary Quantification System of Applied Force in Posteroanteriores Movements Technique (QFPA) is proposed. This equipment aids the physiotherapist students to practice at a long distance. This also allows the teachers to evaluate the applied force in the treatment, and has the possibility to acquire the corresponding data of the therapy session for analysis in real time or for future studies. The students also must use a camera. This is fundamental since allows the teachers to analyze the student hand movements.

\section{REMOTE LABORATORY SYSTEM}

The proposed laboratory system has been designed to integrate all potentials of a physical laboratory to a simple user interface, among with other sub-systems, such as labadministration, instrument operation and hardware management. The design of such a remote laboratory for realtime, internet-based lab-experiments, should consider all aspects of the system, including communication and data flow, as well as instrumentation and hardware control. The developed system is based on a standard client server application software structure. This topology simplifies the architecture and expands the system capabilities. Fig. 1 shows the main functional components of the developed laboratory system. The internet provides the communication infrastructure between the client (therapy laboratory) and the lab server (computer server).

Two of the most important main functional components are the Therapy Laboratory and the Supervised Analysis System. The functional disjointing of these two features allows the uncoupling of the software management (which resides in the laboratory computer) from the physical access to the experimental equipment, as it will be further explained.

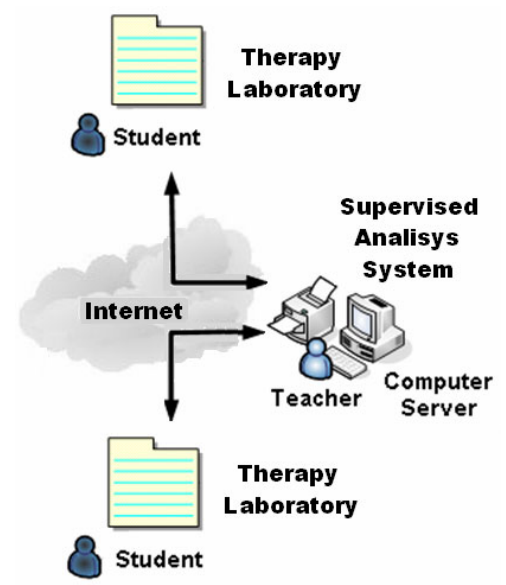

Figure 1. System Functional Components. 


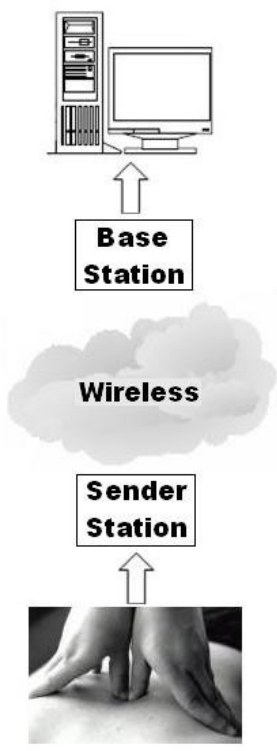

Figure 2. Therapy System Architecture.

The Therapy Laboratory includes features such as access to the physical experiment equipment, measurement system, control and security of the experiments. The Supervised Analysis System includes features such as the user access to the therapy data and the corresponding video information of each individual Therapy Laboratory Management.

The hardware architecture of the experimental therapy system is based on a personal computer, a therapy system including a video camera, and a wireless communication system (Figure 2).

There are two main hardware modules on the Therapy System: the Sender Station and the Base Station. To satisfy the portability characteristics and not to hinder the physiotherapist (student) work, the system has a very light Sender Station that is fastened at the physiotherapist's arm (Figure 3). This station communicates through radio frequency with the Base Station. The Base Station can be positioned anywhere within of the physiotherapist in order to allow him to read the data, without any interference with the technique application.

Each one of the gloves contains two embedded strength sensors which are linked to the Sender Station through three very flexible wires with a Universal Serial Bus (USB) connector. The sensors generate a tension signal, proportional to the applied force by the physiotherapist in the patient treatment. The signal acquisition block does the signal conditioning and configures automatically its gain, as function of the applied force on the patient. The Signal Processing block configures the gain in the Signal Acquisition block, process de data from the glove and from the battery state and prepare these information to the radio frequency emitter. The Power Supply Monitoring block controls the batteries voltage. If the voltage level is lower than a predefined threshold value, the system is not allowed to work. At the end, all the data are sent to the Base Station through the radio frequency (Figure 4).

For the web based application it was used the Matlab Web Server (Figure 5). The Matlab Web Server block manages communications between the Web application and Matlab. The Matlab application is coded in an m-file where additional lines exist to permit bidirectional data transfer between Matlab and html forms. If the m-file generates a graph (.jpeg) this must be copied to a location where the httpd has direct access. In this way the user will send Matlab parameters of a particular test over the Internet and afterwards the obtained results (graphs etc.) are returned to the end user. With this in mind, the users do not need to have MATLAB locally installed or to basically know anything about MATLAB programming.

In the Supervised Analysis system, the computer server receives the information for the entire individual therapy laboratory and has a Matlab application to process and view useful information received from all the applied therapy. The teacher (physiotherapist) analyzes the data and video information from each therapy applied by each student and if necessary tells the student to alter the applied technique. Finally, the application permits to visualize data and graphs corresponding to therapies already accomplished.

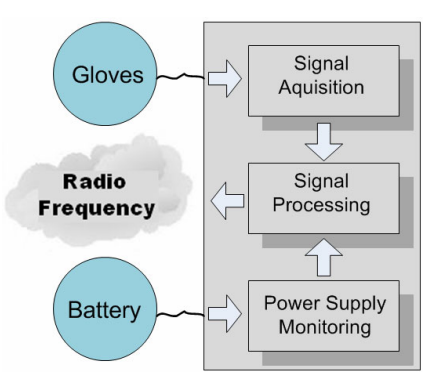

Figure 3. Sender Station blocks diagram.

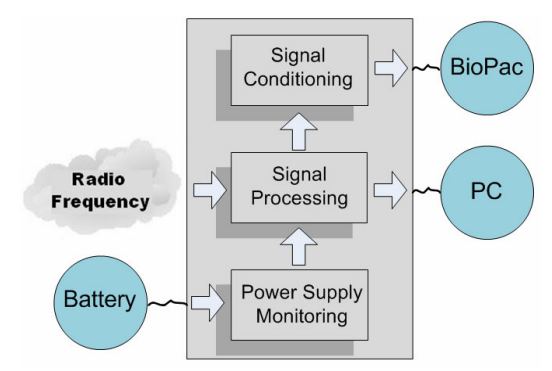

Figure 4. Base Station blocks diagram.

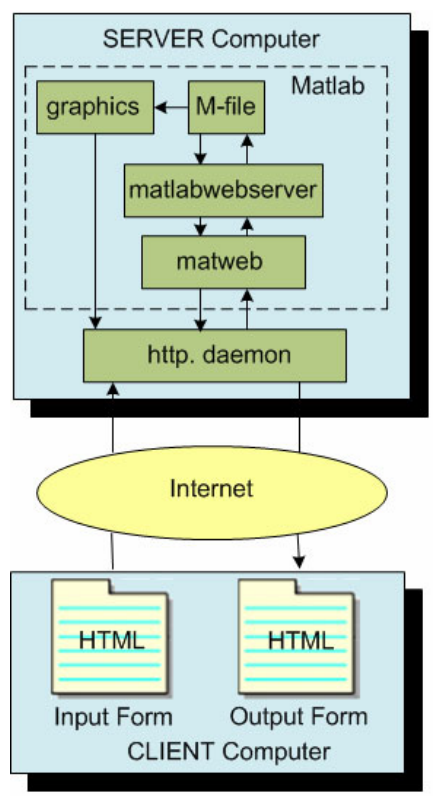

Figure 5. Block diagram of the web based application. 


\section{THERAPY LABORATORY SYSTEM}

Based on recent studies and on the force applied in the therapy technique of the movements SHOVEL, the system was designed based on the following characteristics [16]:

- measurement interval up to $700 \mathrm{~N}$;

- $0,7 \mathrm{~N}$ of resolution;

- high precision;

- high portability;

- high autonomy to guarantee that the system can operate during an enough time interval allowing its use without need to be fed by an external power supply, during the physiotherapy technique;

- friendly use;

- cannot interfere with the application of the physiotherapist's techniques;

- the applied therapy data must be exported to the commercial system named BioPac MP100 [17], already existent in the laboratory of the Superior School of Health of the Polytechnic Institute of Setúbal (Portugal), for scientific and pedagogic objectives.

To implement the strength sensors it was chosen piezoresistive force sensors with the following properties:

- high flexibility;

- sensibility, repeatability, linearity and adequate resolution;

- capability to measure up to $700 \mathrm{~N}$;

- reduced thickness, to allow incorporating the sensors in the gloves, without interfering with the application of the clinical technique.

To conditioning the sensor signals to adequate levels, allowing the analogue to digital signal conversion, it is used an instrumentation amplifier where the gain is controlled to obtain four gain values assuring the adequate resolution for the applied force range. These four gains of the amplifier correspond to the following four force ranges: $[0 \mathrm{~N}, 10 \mathrm{~N}],[10 \mathrm{~N}, 200 \mathrm{~N}],[200 \mathrm{~N}, 400 \mathrm{~N}]$ and $[400 N, 700 N]$ (Figure 6).

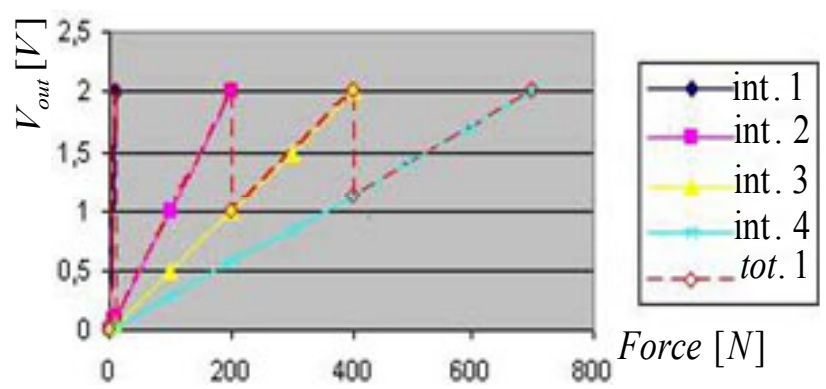

Figure 6. Strength intervals and the corresponding amplifier gains.

The signal sampling is made to the rhythm of 100 samples/second and the radio frequency communication between the Sender Station and the Base Station is made at 2400 bps. These communication structure, allows the PC in the Therapy Laboratory to know which gloves and sensor is in operation.

To assure the correct QFPA operation, the batteries voltage is tested when the system is started up and during the therapy application. If the voltage level is low then just one of the two sensors (thumb or pisiform bone), is used. For these propose, the two ADC channels are verified to measure the applied force in each sensor, being selected the one that reached the initialization level of $10 \mathrm{~N}$. This operation is performed through the ADC mode, Repeat Single Channel (RSC), whose states machine is represented in the Fig. 7.

The developed software on the PC of the Therapy Laboratory presents information in the setup phase and during the therapy treatment. In the therapy, it is presented a graph to visualize, in real time, the force that is being applied. The graph analysis has the normal facilities such as: zoom, selection of an area to be analyzed, and allows read values of the graph with the help of horizontal and vertical cursors (Figure 8).

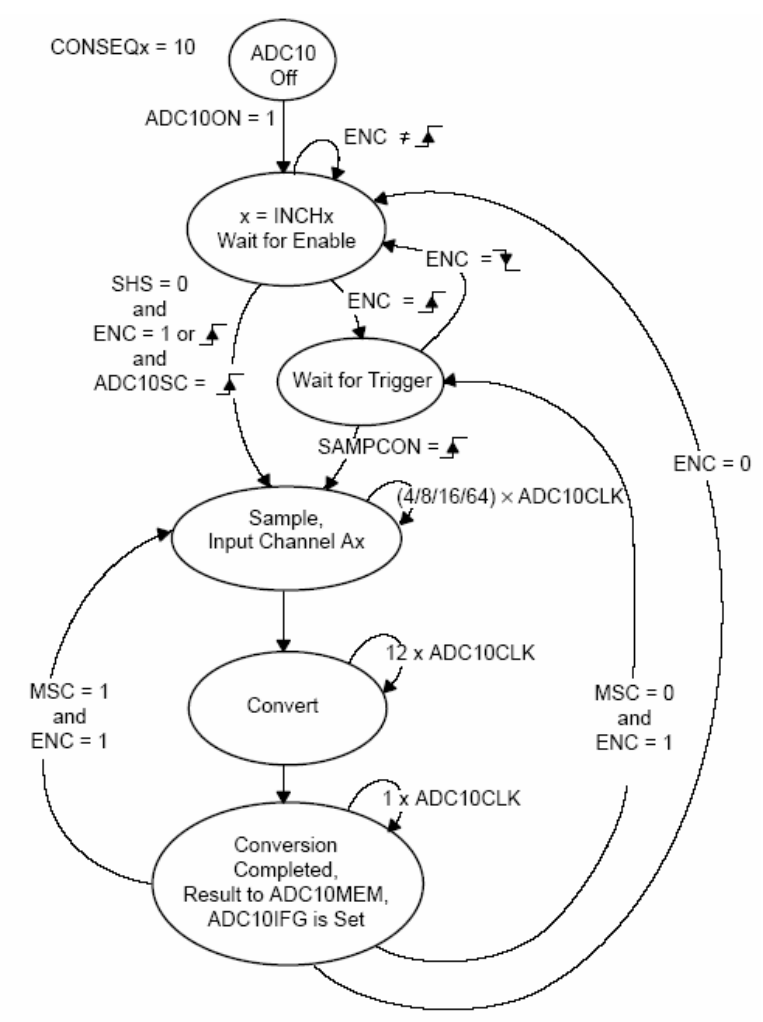

Figure 7. State machine of ADC mode.

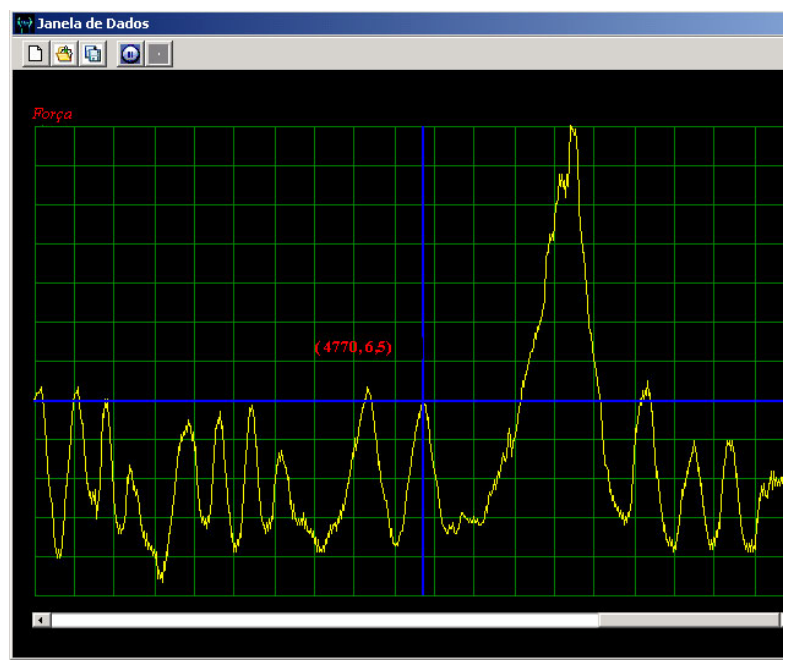

Figure 8. Graph with applied force. 


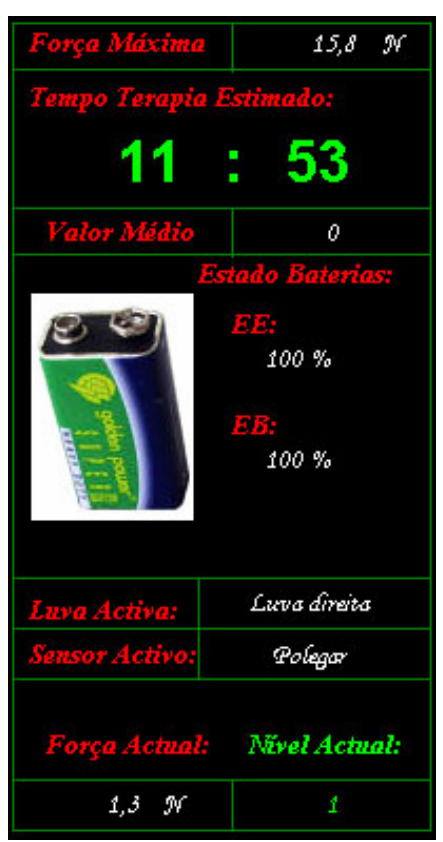

Figure 9. Therapy information.

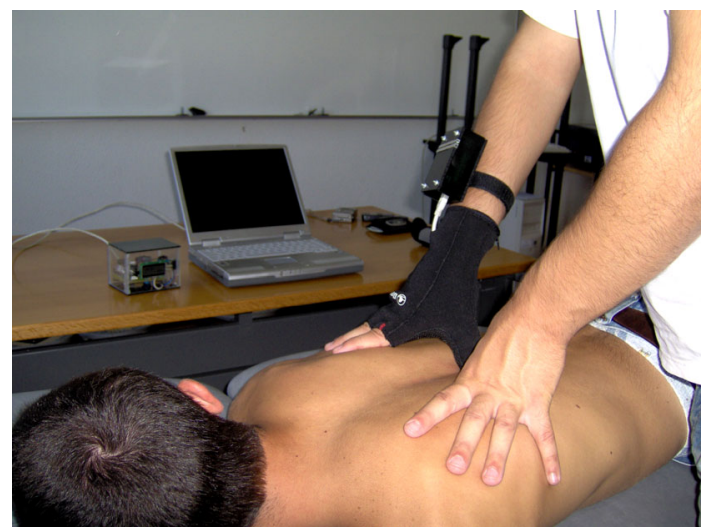

Figure 10. Therapy Laboratory System during the tests.

Fig. 9 shows a screenshot about more information that the teacher has access. In this figure, the teacher can see the maximum force applied for the student, the actual applied force, the estimated time of the therapy and the battery state of charge.

In the performed test to the Therapy Laboratory (Fig. 10), thirty therapies were performed with duration of, approximately, 8 minutes. The correspondent data analysis, has a medium duration of 20 minutes, which showed that the system autonomy is about fifteen hours, without the need of an external power supply.

During the treatment, the student physiotherapist can be positioned in a 3 meters from the Base Station, maintaining good data transmission. Fig. 10 shows a therapy being applied with the thumb sensor.

The obtained results from the QFPA were compared with those achieved from the laboratory equipment used as standard. The comparison results showed that the maximum error is about $\pm 4,5 \%$, which represents a good performance of the proposed Therapy Laboratory System. However, the system performance can still be improved through a better coupling between the sensors and the gloves.

\section{CONCLUSIONS}

In this paper a new remote laboratory environment for manipulative therapy learning was presented. This laboratory aids the physiotherapist students to practice at a long distance. This also allows the teachers to evaluate the applied force in the treatment, and has the possibility to acquire the corresponding data of the therapy session for analysis in real time or for future studies. The implementation of such laboratory uses standard PCs, the popular software package Matlab, gloves with sensors and a camera. The camera allows the teacher to see the student movements.

\section{ACKNOWLEDGMENT}

The authors want to express their appreciation to the students Gustavo Leitão and Marco Francisco of the Engineering Course on Electronics and Computers of the Superior School of Technology of Setúbal (Portugal), about the work developed in the prototype achievement, as well as the collaboration given by the Superior School of Health of the Polytechnic Institute of Setúbal (Portugal).

\section{REFERENCES}

[1] C. Smith, "Introducing eLearning opportunities to School Teachers (k-12) "Getting Your Class Online"," International Journal of The Computer, the Internet and Management, vol. 12, $\mathrm{n}^{\circ} 2$, pp. 8792, May-August, 2004.

[2] I. Gustavsson, "A Remote Laboratory for Electrical Experiments," ASEE Annual Conference Montreal, pp. 11285-11293, June 2002.

[3] S. Hsu, B. Alhalabi, M. Ilyas, "A Java-Based Remote Laboratory for Distance Learning," ICEE 2000 - International Conference on Engineering Education, pp. 1-5, August 2000.

[4] M.E. Macias, I. Mendez, "eLab - Remote Electronics Lab in Real Time," 37th ASEE Annual Frontiers in Education Conference, pp. 1-6, October 2007.

[5] I. Gustavsson, J. Zackrisson, T. Olsson, "Traditional Lab Sessions in a Remote Laboratory for Circuit Analysis," ASEE Annual Conference, pp. 1-6, June 2003.

[6] A. Mohtar, Z. Nedic, J. Machotka, "A Remote Laboratory for Microelectronics Fabrication," 37th ASEE Annual Frontiers in Education Conference, pp. 1-5, October 2008.

[7] J. A. del Alamo, L. Brooks, C. McLean, J. Hardison, G. Mishuris, V. Chang, L. Hui, "The MIT Microelectronics WebLab: a WebEnabled Remote Laboratory for Microelectronics Device Characterization," 2002 World Congress on Networked Learning in a Global Environment, pp. 1-7, May 2002.

[8] Mihaela M. Albu, Keith E. Holbert, Gerald Thomas Heydt, Sorin Dan Grigorescu, Vasile Trusca, "Embedding Remote Experimentation in Power Engineering Education," IEEE Transactions on Power Systems, vol. 19, no 1, pp. 139-143, February 2004. (doi:10.1109/TPWRS.2003.821020)

[9] V. Fernão Pires, L. Sousa Martins, Tito G. Amaral, Rui Marçal, Ricardo Rodrigues, Manuel M. Crisóstomo, "Distance Learning Power System Protection Based on Testing Protective Relays," IEEE Transactions on Industrial Electronics, vol. 55, $\mathrm{n}^{\circ} 6, \mathrm{pp}$. 2433-2438, June 2008.

[10] R. Marín, P. J. Sanz, P. Nebot, R. Wirz, “A Multimodal Interface to Control a Robot Arm via the Web: A Case Study on Remote Programming," IEEE Transactions on Industrial Electronics, vol. 52, no 6, pp. 1506-1520, December 2005. (doi:10.1109/ TIE.2005.858733)

[11] R. Šafaric, M. Truntic, D. Hercog G. Pacnik, "Control and robotics remote laboratory for engineering education," iJOE International Journal on Online Engineering, vol. 1, no 1, pp. 1-8, 2005.

[12] M. Casini, D. Prattichizzo, A. Vicino, "The Automatic Control Telelab," IEEE Control Systems Magazine, pp. 36-44, June 2004. (doi:10.1109/MCS.2004.1299531) 
[13] A. Valera, J. L. Diez, M. Valles, P. Albertos, "Virtual and Remote Control Laboratory Development," IEEE Control Systems Magazine, pp. 35-39, February 2005. (doi:10.1109/MCS.2005.1388798)

[14] R. Jurkonis, V. Marozas and A. Lukoševičius, "Ultrasound Medical Diagnostics Laboratory for Remote Learning in EVICAB*Campus," Innovative Techniques in Instruction Technology, E-learning, E-assessment, and Education, Springer, pp. 453-456, 2008.

[15] Geoff Maitland, Elly Hengeveld, Kevin Banks, Kay English, "Maitland's Vertebral Manipulation", 6th edition, 2001.

[16] Garry Allison, Stephen Edmonston, Kari Kiviniemi, Helen Lanigan, AnineVibe Simonsen e Susanne Walcher, "Influence of standardized mobilization on the posteroanterior stiffness of the lumbar spine in asymptomatic subjects", Physiotherapy Research International, Volume 6, Issue 3, pp. 145-156, 2001. (doi:10.1002/pri.223)

[17] Biopack M100 Workstation, Biopac Systems, Inc, http://www.lintoninst.com.uk/mp100_main.htm.

\section{AUTHORS}

O. P. Dias is with the Electrical Engineering Department, Setúbal School of Technology, Polytechnic Institute of Setúbal, IPS, CESET, INESC-ID Lisboa, 2910-761 Portugal (e-mail: pdias@est.ips.pt).

T. G. Amaral, is with the Electrical Engineering Department, Setúbal School of Technology, Polytechnic Institute of Setúbal, IPS, CESET, ISR - Pólo de Coimbra, 2910-761 Portugal (e-mail: tamaral@est.ips.pt).

V. Fernão Pires is with the Electrical Engineering Department, Setúbal School of Technology, Polytechnic Institute of Setúbal, IPS, CIEEE Lisboa, 2910-761 Portugal (e-mail: vpires@est.ips.pt).

Manuscript received 4 November 2008. Published as submitted by the authors. 